\title{
ON LOCAL SMOOTHING PROBLEMS AND STEIN'S MAXIMAL SPHERICAL MEANS
}

\author{
CHANGXING MIAO, JIANWEI YANG AND JIQIANG ZHENG
}

\begin{abstract}
It is proved that the local smoothing conjecture for wave equations implies certain improvements on Stein's analytic family of maximal spherical means. Some related problems are also discussed.
\end{abstract}

\section{INTRODUCTION}

For $\alpha>0$, we let $m_{\alpha}(x)=\Gamma(\alpha)^{-1}\left(1-|x|^{2}\right)_{+}^{\alpha-1}$ where $x \in \mathbb{R}^{n}, \Gamma(\alpha)$ is the Gamma function and $r_{+}$is a homogeneous distribution defined to be $r$ when $r>0$, and equal to 0 if $r \leq 0$. Denote by $m_{\alpha, t}(x)=m_{\alpha}(x / t) t^{-n}$ for $t>0$ and define

$$
\mathscr{M}_{t}^{\alpha} f(x)=\left(f * m_{\alpha, t}\right)(x),
$$

initially for $f \in C_{0}^{\infty}\left(\mathbb{R}^{n}\right)$.

These averaging operators are defined a priori only for real positive $\alpha$. However, if we recall the Fourier transform of $m_{\alpha}$

$$
\widehat{m}_{\alpha}(\xi)=\pi^{-\alpha+1}|\xi|^{-\frac{n}{2}-\alpha+1} \mathcal{J}_{\frac{n}{2}+\alpha-1}(2 \pi|\xi|),
$$

where $\mathcal{J}_{m}(x)$ is the Bessel function of order $m$ ( see [18] or Appendix), and notice that $\operatorname{Re} \alpha \leq 0$ is allowed in the Bessel functions in (1.2) by means of analytic continuation, we can extend the notion of (1.1) to include complex $\alpha$ via Fourier transform

$$
\widehat{\mathscr{M}_{t}^{\alpha} f}(\xi)=\widehat{m}_{\alpha}(\xi t) \widehat{f}(\xi), f \in C_{0}^{\infty}\left(\mathbb{R}^{n}\right) .
$$

It is also important to remark that $\widehat{m}_{\alpha}(0)$ is finite and $\widehat{m}_{\alpha}(\xi)$ is smooth near the origin. For details on these standard facts, we refer to [16] and Theorem 4.15, Chap IV in [18].

It is not hard to see that in the sense of distribution

$$
\lim _{\alpha \rightarrow 0+} \frac{1}{\Gamma(\alpha)} t_{+}^{\alpha-1}=\delta(t)
$$

Date: July 28, 2017.

2000 Mathematics Subject Classification. 42B25, 42B20.

Key words and phrases. Maximal spherical means, local smoothing, wave equation, oscillatory integral. 
where $\delta(t)$ denotes the Dirac distribution at zero. In particular, for $f \in$ $C_{0}^{\infty}\left(\mathbb{R}^{n}\right)$, we have by the co-area formula

$$
\mathscr{M}_{t}^{0} f(x)=\lim _{\alpha \rightarrow 0+} \mathscr{M}_{t}^{\alpha} f(x)=c_{n} \int_{S^{n-1}} f(x+y t) d \sigma(y),
$$

where $c_{n}$ is a constant depending only on $n, S^{n-1}$ denotes the standard unit sphere in $\mathbb{R}^{n}$ and $d \sigma$ corresponds to the normalized surface measure induced from Lebesgue measure on $\mathbb{R}^{n}$. In what follows, we call (1.4) the spherical means of $f$.

Let $M^{\alpha}$ be the maximal operator associated to $\mathscr{M}_{t}^{\alpha}$ defined as

$$
M^{\alpha}(f)(x)=\sup _{t>0}\left|\mathscr{M}_{t}^{\alpha} f(x)\right| .
$$

In [16], Stein proved when $n \geq 3$, one has

$$
\left\|M^{\alpha}(f)\right\|_{L^{p}\left(\mathbb{R}^{n}\right)} \leq A_{p, \alpha}\|f\|_{L^{p}\left(\mathbb{R}^{n}\right)}, 0<A_{p, \alpha}<+\infty,
$$

under the following condition

$$
\operatorname{Re} \alpha>1-n+\frac{n}{p}, \quad \text { if } \quad 1<p \leq 2,
$$

or

$$
\operatorname{Re} \alpha>\frac{2-n}{p}, \quad \text { if } \quad 2 \leq p \leq \infty,
$$

where the two dimensional case was left open since then the necessary condition $p>2$ for $\alpha=0$ eliminates the use of $L^{2}$ argument based on Plancherel's theorem. The above two admissible relations for $\alpha$ and $p$ are summarized when $n \geq 3$ in Figure 1, where the relation (1.8) corresponds to the dotted segment $O B$.

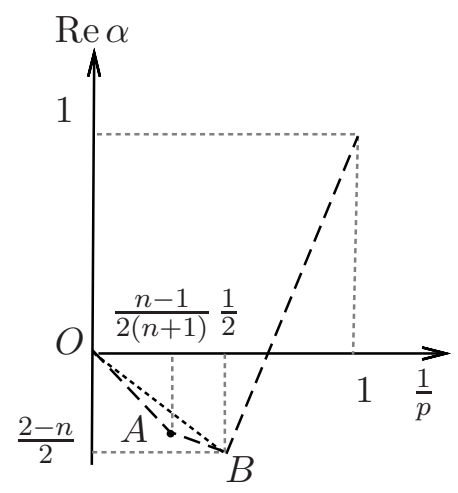

FiguRe 1.

The condition (1.8) is not optimal. An important problem is how to extend this range for $\alpha$ and $p$, where $2<p \leq \infty$. In this paper, we are interested in investigating on this problem and show that it is possible to 
get certain improvement by using the local smoothing estimate of linear wave equations. More precisely, our main theorem reads

Theorem 1.1. Suppose $n \geq 2$ and for $p \geq 2$ denote by

$$
\varepsilon(p, n)=\min \left\{\frac{n-1}{4}+\frac{n-3}{2 p}, \frac{n-1}{p}\right\} .
$$

Then, (1.6) is valid for all $\alpha \in \mathbb{C}$ and $p>2$ such that $\operatorname{Re} \alpha>-\varepsilon(p, n)$.

Remark 1.2. Compared to (1.8), there is a $1 / p$-downwards extension for the range of $\operatorname{Re} \alpha$ so that (1.6) is valid for $p \geq p_{n}$ where $p_{n}=2 \frac{n+1}{n-1}$, and there is a $\frac{n-1}{2}\left(\frac{1}{2}-\frac{1}{p}\right)$-improvement for $2<p<p_{n}$. This is indicated in Figure 1 by the dashed line segments $O A$ and $A B$.

The proof of Theorem 1.1 relies on the recent results concerning Sogge's local smoothing conjecture for wave equations. Let $u(t, x)$ be the solution to the Cauchy problem

$$
\left\{\begin{array}{c}
\left(\partial_{t}^{2}-\Delta\right) u(x, t)=0,(x, t) \in \mathbb{R}^{n} \times \mathbb{R}, \\
u(x, 0)=f(x), \partial_{t} u(x, 0)=g(x) .
\end{array}\right.
$$

It is conjectured in [14] that for $n \geq 2$ and $p \geq \frac{2 n}{n-1}$, one has

$$
\|u\|_{L^{p}\left(\mathbb{R}^{n} \times[1,2]\right)} \leq C\left(\|f\|_{W^{\gamma, p}\left(\mathbb{R}^{n}\right)}+\|g\|_{W^{\gamma-1, p\left(\mathbb{R}^{n}\right)}}\right), \gamma>\frac{n-1}{2}-\frac{n}{p},
$$

where $W^{\gamma, p}$ represents the usual inhomogeneous Sobolev space. Indeed, the reader will realize that what we proved below is nothing but the fact that (1.11) implies the above downwards extension of the range of $\alpha$, and Theorem 1.1 follows simply from the best knowledge on $p$ for which (1.11) is true.

Some pioneer results on (1.11) with loss of derivatives appeared chronologically in [14, 9, 10, 15]. Their arguments involve mainly, among other things, orthogonality, square-function estimates and certain variable coefficient versions of Kakeya-Nykodim type maximal inequalities, as can be found in [15] and Chapter $\mathrm{X}$ in [17].

In [20], by proving a sharp decoupling inequality, Wolff first obtained (1.11) in dimension two for all $p>74$. The higher dimensional counterpart with $n \geq 3$ was established in [8] for $p>\min \left\{2+\frac{8}{n-3}, 2+\frac{32}{3 n-7}\right\}$, which was improved later in [4] to $p>2+\frac{8}{n-2} \cdot \frac{2 n+1}{2(n+1)}$. The best result is obtained in a very recent work [3], where Bourgain and Demeter proved (1.11) is true for

$$
p \geq \frac{2(n+1)}{n-1}, n \geq 2 \text {. }
$$


Their argument is based on the techniques developed in a series of works on the decoupling inequalities. We refer to [3] for more references and comments on this issue.

If we use interpolation, we have

$$
\|u\|_{L^{p}\left(\mathbb{R}^{n} \times[1,2]\right)} \leq C\|f\|_{W^{\gamma-1, p}\left(\mathbb{R}^{n}\right)},
$$

for $2<p \leq \infty$ and $\gamma>\gamma(p, n)$, where

$$
\gamma(p, n)=\max \left\{\frac{n-1}{2}\left(\frac{1}{2}-\frac{1}{p}\right), \frac{n-1}{2}-\frac{n}{p}\right\} .
$$

It is (1.12) and (1.13) that corresponds to the improvement upon the relation of $\operatorname{Re} \alpha$ and $p$ obtained in Theorem 1.1 .

Remark 1.3. When $p<\frac{2 n}{n-1}$, there is no additional $1 / p$ local smoothing for wave equations, nor for more general Fourier integral operators satisfying cinematic curvature conditions, as pointed out in [15].

Let us turn back to the spherical means and give some historical remarks. In the two dimensional case when $\alpha=0$, the $L^{p}$-boundedness of the circular maximal operator (1.5) for $p>2$ was, ten years around after [16, finally established by Bourgain [1]. Later, this result concerning circular maximal means was generalized to planar convex curves in [2]. This result was extended to the variable coefficient version by Sogge in [14, where it is shown that the translation invariance property of the curves are not essential. However, to ensure a "dynamic" condition, an assumption on cinematic curvature condition is required. See [14, 15] and [17] for more details. Further more, results of this kind are extended to some possible $L^{q}-L^{p}$ estimate for certain $q>p$ by Schlag [12] using Kolasa-Wolff's geometric/combinatorial method [7]. This result was recovered and generalized to the higher dimensional counterparts as well as to its variable coefficient cases by Schlag-Sogge in [13] using partial local smoothing estimates.

If one removes the restriction on $\alpha=0$ and allows $\alpha$ to take complex values, (1.6) was strengthened when $n=2$ by Mockenhaupt, Seeger and Sogge in 9] for all $\operatorname{Re} \alpha>-\varepsilon(p) / 2$, which extends (1.8) for

$$
\varepsilon(p)= \begin{cases}\frac{1}{2}-\frac{1}{p}, & 2<p<4 ; \\ \frac{1}{p}, & 4 \leq p<\infty .\end{cases}
$$

As far as we know, this is the first work connecting the maximal circular means with the local smoothing problems of wave equations, or more generally, Fourier integral operators satisfying Sogge's cinematic curvature condition.

Around the maximal operator (1.5), there are two possible ways of doing extensions. One is to consider the variable coefficient version for (1.6) with 
$\alpha=0$. These results are investigated in [15, including both $n \geq 3$ and $n=2$, where the two cases are treated separately by means of Fourier integral operators. Another direction is to extend the admissible relation for $\alpha$ and $p$ in (1.8) for (1.6) as far as possible. Thus, it follows a natural question whether one may combine the two issues together by extending (1.6) to the non-translation-invariance setting with $\alpha \neq 0$.

Before ending up this section, let us state an application of Theorem 1.1 to the wave equations.

Corollary 1.4. Suppose $n \geq 4$ and let $u(x, t)$ be the solution of the problem (1.10) with $f(x) \equiv 0$. If $p \in\left(\frac{2 n}{n+1}, \frac{2(n-1)}{n-3}\right)$ and $g \in L^{p}\left(\mathbb{R}^{n}\right)$, then we have

$$
\lim _{t \rightarrow 0} \frac{u(x, t)}{t}=g(x)
$$

for almost every $x \in \mathbb{R}^{n}$.

Proof. As in [16], if we take $\alpha=\frac{3-n}{2}$ and $c_{n}=\frac{1}{2} \pi^{-\frac{n}{2}-\frac{1}{2}}$, then

$$
u(x, t)=c_{n} t \mathscr{M}_{t}^{\alpha}(g)(x),
$$

solves the Cauchy problem of the wave equation (1.10) with $f(x) \equiv 0$. We refer to a straightforward interpretation on (1.16) in Appendix. As a consequence of (1.7) and Theorem 1.1, (1.6) is true for

$$
p \in\left(\frac{2 n}{n+1}, 2\right] \bigcup\left[\frac{2(n+1)}{n-1}, \frac{2(n-1)}{n-3}\right) .
$$

Hence, we have (1.6) for $p \in\left(\frac{2 n}{n+1}, \frac{2(n-1)}{n-3}\right)$ by interpolation. From this, we conclude (1.15) for the same range of $p$.

Remark 1.5. Notice that the above almost everywhere convergence result (1.15) was proved for $g \in L^{p}\left(\mathbb{R}^{n}\right)$, where $\frac{2 n}{n+1}<p<\frac{2(n-2)}{(n-3)}$ for $n \geq 3$ in in [16]. This above corollary slightly improves this result when $n \geq 4$.

To end up this section, we indicate that it is commented in [17 that the optimal results for $p>2$ and $n \geq 2$ "are still a mystery". Although we can show that under the assumption of sharp local smoothing estimate for wave equations, the admissible range as for (1.6) is enlarged as in Remark 1.2, we do not know whether this is already optimal or not.

This paper is organized as follows. In Section 2, we prove Theorem 1.1, where the proof is divided into four steps. Section 3 is devoted to some remarks and comments for further study around this topic. In Appendix, we clarify certain identities used in the introduction. Although these are rather standard facts, we include them for the convenience of reading. 
Acknowledgments: The authors thank the referees for spending their time reading and comments which improve this paper a lot. This work was supported in part by the National Natural Science Foundation of China under grant No.11231006, and No.11671047. C. Miao was also supported by Beijing Center for Mathematics and Information Interdisciplinary Sciences. J. Yang was supported by ERC Advanced Grant No. 291214 BLOWDISOL . J. Zheng was partly supported by the European Research Council, ERC2012-ADG, project number 320845 : Semi-Classical Analysis of Partial Differential Equations.

\section{Proof of Theorem 1.1}

This section is devoted to the proof of our main theorem. We start with an outline of the argument. First, we prove a truncated maximal function where the supremum is taken over $t \in[1,2]$. To dominate the supremum, we use Sobolev embedding $W^{\beta, p}(I) \hookrightarrow L^{\infty}([1,2])$ where $I$ is a suitable compact interval containing $[1,2]$ and $\beta>\frac{1}{p}$. This enforces us to define the fractional order derivative of $\mathscr{M}_{t}^{\alpha}(f)(x)$ in $t$-variable. For this, we will, after applying Littlewood-Paley decomposition to $f$, write for each $j \geq 1$

$$
\left|\partial_{t}\right|^{\beta} \mathscr{M}_{t}^{\alpha}\left(\Delta_{j} f\right)(x)
$$

as an integration operator, where the distributional kernel can be represented by the difference of the two following oscillatory integrals

$$
\begin{aligned}
& \mathcal{I}_{j}(x, y)=\iint a_{1, j}(\tau, s, \xi) e^{2 \pi i \phi_{1}(x, y, t ;, \xi, \tau, s, \theta)} d \theta d s d \tau d \xi, \\
& \mathcal{J}_{j}(x, y)=\iint a_{2, j}(\tau, s, \xi) e^{2 \pi i \phi_{2}(x, y, t ;, \xi, \tau, s, r)} d r d s d \tau d \xi,
\end{aligned}
$$

where $a_{1, j}(\tau, s, \xi)$ and $a_{2, j}(\tau, s, \xi)$ are two appropriate symbols and the two phase functions read

$$
\begin{aligned}
& \phi_{1}(x, y, t ;, \xi, \tau, s, \theta)=(x-y) \cdot \xi+(t-s) \tau+s|\xi| \sin \theta-\theta \varpi / 2 \pi, \\
& \phi_{2}(x, y, t ;, \xi, \tau, s, r)=(x-y) \cdot \xi+(t-s) \tau+i(|\xi| s \sinh r+\varpi r / 2 \pi) .
\end{aligned}
$$

Observe that $\phi_{1}$ has critical points in the $s$-variable only if $\tau \approx|\xi| \sin \theta$. This suggests us to localize the frequency of time by means of truncating $\tau$ to the low frequency. Combined with Littlewood-Pelay decomposition and scaling, the temporal regularity is transferred to the spacial derivatives. In the proof, method of stationary phase and Schläfli's integral representation of Bessel functions in [19]: for $r \in \mathbb{R}^{+}$and $k>-\frac{1}{2}$,

$$
\begin{aligned}
\mathcal{J}_{k}(r) & =\frac{1}{2 \pi} \int_{-\pi}^{\pi} e^{i r \sin \theta} e^{-i \theta k} d \theta-\frac{\sin (k \pi)}{\pi} \int_{0}^{\infty} e^{-(r \sinh (s)+k s)} d s \\
& :=\tilde{J}_{k}(r)-E_{k}(r)
\end{aligned}
$$

play a central role. The former eliminates the error terms in the following arguments while the latter gives rise to the half wave operator in Step 3 so that we may involve the sharp local smoothing estimate. From now on, we 
always assume $\operatorname{Re} \alpha<0$ since this is the interesting situation. At the end of the proof, we will eliminate the restriction on $t \in[1,2]$ by a standard trick. Now, let us turn to the rigorous proof.

The proof of Theorem 1.1. We take a function $\varphi \in C_{0}^{\infty}(\mathbb{R})$ such that $\operatorname{supp} \varphi \subset[1 / 2,2]$ to form a partition of unity $\sum \varphi\left(2^{-j} s\right)=1$, where $s \in$ $\mathbb{R} \backslash\{0\}$ and the summation is taken over all integers $j \in \mathbb{Z}$. For $\xi \in \mathbb{R}^{n}$, we define

$$
\begin{gathered}
\varphi_{j}(\xi)=\varphi\left(2^{-j}|\xi|\right), \text { for } j \geq 1, \\
\varphi_{0}(\xi)=1-\sum_{j=1}^{\infty} \varphi_{j}(\xi),
\end{gathered}
$$

and write $\widehat{\Delta_{j} f}(\xi)=\varphi_{j}(\xi) \widehat{f}(\xi)$ for $j \geq 0$, where $\Delta_{j}$ denotes the well-known Littlewood-Pelay's projector. To use the reproducing property, we will also use $\tilde{\varphi}$ to represent a smooth positive function identical to one on the support of $\varphi$ and vanishing outside the interval $(1 / 4,4)$. We then define $\tilde{\Delta}_{j} f(x)$ via $\widehat{\widetilde{\Delta}_{j} f}(\xi)=\tilde{\varphi}\left(2^{-j}|\xi|\right) \widehat{f}(\xi)$. Notice that in the following argument, we may assume $\alpha$ is real and $\alpha<0$ whereas the general case for complex $\alpha$ follows the same reasoning. The proof is divided into four steps.

Step 1. In this step, we show the low frequency part is well behaved, namely there is some constant $C>0$ such that

$$
\left\|\sup _{1<t<2}\left|\mathscr{M}_{t}^{\alpha}\left(\Delta_{0} f\right)(x)\right|\right\|_{L^{p}\left(\mathbb{R}^{n}\right)} \leq C\|f\|_{L^{p}\left(\mathbb{R}^{n}\right)} .
$$

In fact, it is immediate once we have

$$
\sup _{1<t<2}\left|\mathscr{M}_{t}^{\alpha}\left(\Delta_{0} f\right)(x)\right| \leq C M_{H L}(f)(x),
$$

where $M_{H L}$ denotes the standard Hardy-Littlewood maximal function. This is because $\widehat{m}_{\alpha}(\xi t) \varphi_{0}(\xi)$ is smooth and supported in $|\xi| \leq 2$. Thus (2.2) follows immediately from the $L^{p}$ boundedness of $M_{H L}$ for any $p>1$.

Step 2. In this step, we single out the main contribution of $\mathscr{M}_{t}^{\alpha}\left(\Delta_{j} f\right)(x)$ for each $j$. Choose a smooth positive function $\chi$ identical to one in a neighborhood of $[1,2]$ and vanishing outside $(1 / 2,4)$. Taking also a smooth positive function $\chi_{0}(\tau)$, identical to one on the interval $[-4,4]$ and zero outside $(-8,8)$, we set $\chi_{I_{j}}(\tau)=\chi_{0}\left(2^{-j} \tau\right)$. We consider the space-time Fourier transform of $\chi(t) \mathscr{M}_{t}^{\alpha}\left(\Delta_{j} f\right)(x)$ as calculated below

$$
\begin{aligned}
& \mathcal{F}_{(x, t) \rightarrow(\xi, \tau)}\left(\chi(t) \mathscr{M}_{t}^{\alpha}\left(\Delta_{j} f\right)(\cdot)\right)(\xi, \tau) \\
= & |\xi|^{-\varpi} \varphi_{j}(\xi) \widehat{f}(\xi)\left[\int_{-\pi}^{\pi} \widehat{\chi_{1}}(\tau-|\xi| \sin \theta) e^{-i \theta \varpi} d \theta-\int_{0}^{\infty} \widehat{\chi_{2}}(\tau-i|\xi| \sinh s) e^{-\varpi s} d s\right],
\end{aligned}
$$

where $\varpi=\frac{n}{2}+\alpha-1$, and

$$
\chi_{1}(t)=\frac{1}{2 \pi^{\alpha}} \chi(t) t^{-\varpi}, \chi_{2}(t)=\chi(t) t^{-\varpi} \frac{\sin \varpi \pi}{\pi^{\alpha}} .
$$


We note that $\widehat{\chi}_{2}(\tau)$ can be extended to the upper half complex plane $\{\tau \in$ $\mathbb{C}: \operatorname{Im} \tau \geq 0\}$ as an analytic function since $\chi_{2}$ is smooth and compactly supported.

Thus, we may define for $j \geq 1$,

$$
\left|\partial_{t}\right|^{\beta}\left(\chi(t) \mathscr{M}_{t}^{\alpha}\left(\Delta_{j} f\right)(x)\right)=\iint e^{2 \pi i(x \cdot \xi+t \tau)} a_{j}(\tau, \xi) \widehat{f}(\xi) d \tau d \xi
$$

and

$$
\mathscr{F}_{j} f(x, t)=\iint e^{2 \pi i(x \cdot \xi+t \tau)} \chi_{I_{j}}(\tau) a_{j}(\tau, \xi) \widehat{f}(\xi) d \tau d \xi,
$$

with an amplitude $a_{j}(\tau, \xi)=a_{j, 1}(\tau, \xi)-a_{j, 2}(\tau, \xi)$, where

$$
\begin{aligned}
& a_{j, 1}(\tau, \xi)=|\xi|^{-\varpi} \varphi_{j}(\xi)|\tau|^{\beta} \int_{-\pi}^{\pi} \widehat{\chi}_{1}(\tau-|\xi| \sin \theta) e^{-i \theta \varpi} d \theta, \\
& a_{j, 2}(\tau, \xi)=|\xi|^{-\varpi} \varphi_{j}(\xi)|\tau|^{\beta} \int_{0}^{\infty} \widehat{\chi_{2}}(\tau-i|\xi| \sinh s) e^{-\varpi s} d s .
\end{aligned}
$$

In what follows, we consider the contributions of $a_{j, 1}$ and $a_{j, 2}$, separately.

- Estimation on the first error term. Write

$$
\mathscr{F}_{j, 1}(f)(x, t)=\iint e^{2 \pi i(x \cdot \xi+t \tau)} \tilde{a}_{j, 1}(\tau, \xi) \widehat{f}(\xi) d \tau d \xi
$$

where $\tilde{a}_{j, 1}(\tau, \xi)=\chi_{I_{j}}(\tau) a_{j, 1}(\tau, \xi)$ and consider the error term

$$
\mathscr{R}_{j, 1}(f)(x, t):=\iint e^{2 \pi i(x \cdot \xi+t \tau)} a_{j, 1}(\tau, \xi) \widehat{f}(\xi) d \tau d \xi-\mathscr{F}_{j, 1}(f)(x, t) .
$$

We have

$$
\mathscr{R}_{j, 1}(f)(x, t)=\int K_{j}(x, t, y) \tilde{\Delta}_{j} f(y) d y
$$

where the distributional kernel is given by

$$
K_{j}(x, t, y)=\int_{-\pi}^{\pi} \mathscr{K}_{j}(x, t, y ; \theta) d \theta
$$

and $\mathscr{K}_{j}(x, t, y ; \theta)$ may take the following form for any prefixed $N>0$

$$
\mathscr{K}_{j}(x, t, y ; \theta)=e^{-i \theta \varpi} \int\left(1-\chi_{I_{j}}(\tau)\right) \mathscr{H}_{j}(\tau, \theta, N ; x, y) \frac{|\tau|^{\beta} e^{2 \pi i t \tau} d \tau}{\left(1+|\tau|-2^{j+1}\right)^{N}},
$$

provided that

$$
\mathscr{H}_{j}(\tau, \theta, N ; x, y)=\left(1+|\tau|-2^{j+1}\right)^{N} \int \widehat{\chi_{1}}(\tau-|\xi| \sin \theta) \varphi_{j}(\xi) \frac{e^{2 \pi i(x-y) \cdot \xi}}{|\xi|^{\varpi}} d \xi .
$$

Next, from integration by parts, we can seek a $C_{N}>0$ such that

$$
\left|\mathscr{H}_{j}(\tau, \theta, N ; x, y)\right| \leq C_{N} 2^{-j(\varpi-n)} \cdot 2^{100 j n}\left(1+2^{j}|x-y|\right)^{-100 n} .
$$

Therefore, we have

$$
\left\|\mathscr{R}_{j, 1}(f)(\cdot, t)\right\|_{L_{x}^{p}\left(\mathbb{R}^{n}\right)} \leq C_{N}^{\prime} 2^{-j \frac{N}{2}}\left\|\tilde{\Delta}_{j} f\right\|_{p}, \text { when } \frac{1}{2} \leq t \leq 4,
$$


for large $N$ and some $C_{N}^{\prime}>0$.

- Estimation on the second error term. We will see

$$
\mathscr{F}_{j, 2}(f)(x, t):=-\iint e^{2 \pi i(x \cdot \xi+t \tau)} \tilde{a}_{j, 2}(\tau, \xi) \widehat{f}(\xi) d \tau d \xi
$$

carries the main contribution from $a_{j, 2}$, where $\tilde{a}_{j, 2}(\tau, \xi)=\chi_{I_{j}}(\tau) a_{j, 2}(\tau, \xi)$ and $\chi_{I_{j}}(\tau)$ is defined the same as before. Since the argument are very similar, we only sketch the proof below with some necessary remarks.

Similar to $\mathscr{R}_{j, 1}$, there is an error term

$$
\mathscr{R}_{j, 2}(f)(x, t)=\int_{\mathbb{R}^{n}} L_{j}(x, t, y) \tilde{\Delta}_{j} f(y) d y,
$$

with

$$
\begin{aligned}
L_{j}(x, t, y)=\int_{0}^{\infty} d s & \int\left(1-\chi_{I_{j}}(\tau)\right)|\tau|^{\beta} e^{2 \pi i t \tau} e^{-\varpi s} d \tau \\
& \times \int_{\mathbb{R}^{n}} \varphi_{j}(\xi) \widehat{\chi_{2}}(\tau-i|\xi| \sinh s) \frac{e^{2 \pi i(x-y) \cdot \xi}}{|\xi|^{\varpi}} d \xi .
\end{aligned}
$$

Noting that $\tau$ is restricted to $|\tau|>2^{j+2}$, we may insert $\left(1+|\tau|-2^{j+1}\right)^{-N}$ into the integration and changing variables $\xi \rightarrow 2^{j} \xi$. Then, using integration by parts, we can control

$$
\widehat{\chi}_{2}\left(\tau-i 2^{j}|\xi| \sinh s\right)=\int \chi_{2}(r) e^{-2 \pi\left(2^{j}|\xi| \sinh s\right) r} e^{-2 \pi i r \tau} d r
$$

by

$$
\left\|\chi_{2}^{\left(N^{\prime}\right)}\right\|_{L^{1}}(1+|\tau|)^{-N^{\prime}} e^{-\pi 2^{j-1}|\xi| \sinh s}, \text { with } s>0,
$$

so that $\left(1+|\tau|-2^{j+1}\right)^{N}$ is absorbed by $\widehat{\chi}_{2}\left(\tau-i 2^{j}|\xi| \sinh s\right)$. Again, we also have

$$
\left\|\mathscr{R}_{j, 2}(f)(\cdot, t)\right\|_{L_{x}^{p}\left(\mathbb{R}^{n}\right)} \leq C_{N}^{\prime} 2^{-j \frac{N}{2}}\left\|\tilde{\Delta}_{j} f\right\|_{p}, \text { when } \frac{1}{2} \leq t \leq 4,
$$

for large $N$ and some $C_{N}^{\prime}>0$.

Since $\mathscr{F}_{j}(f)=\mathscr{F}_{j, 1}(f)+\mathscr{F}_{j, 2}(f)$, it remains to obtain the right estimate for the main term $\mathscr{F}_{j}(f)$.

Step 3. In this step, we evaluate the space-time $L^{p}\left(\mathbb{R}^{n} \times \mathbb{R}\right)$ norm of $\mathscr{F}_{j}(f)(x, t)$ by means of local smoothing. Denote by $f_{j}(x)=f\left(2^{-j} x\right)$ and change variables $(\tau, \xi) \rightarrow\left(2^{j} \tau, 2^{j} \xi\right)$ to get

$$
\mathscr{F}_{j}(f)(x, t)=\iint e^{2 \pi i 2^{j}(x \cdot \xi+t \tau)} 2^{j} a_{j}\left(2^{j} \tau, 2^{j} \xi\right) \widehat{f}_{j}(\xi) d \xi d \tau
$$

where

$$
2^{j} a_{j}\left(2^{j} \tau, 2^{j} \xi\right)=2^{-j(\varpi-\beta)} \tilde{a}_{j}(\tau, \xi)
$$

$$
\tilde{a}_{j}(\tau, \xi)=2^{j} \cdot 2 \pi|\xi|^{-\varpi} \varphi(\xi)|\tau|^{\beta} \chi_{0}(\tau) \int \tilde{\chi}(s) \mathcal{J}_{\varpi}\left(2 \pi \cdot 2^{j} s|\xi|\right) e^{-2 \pi i 2^{j} \tau s} d s .
$$


Using the asymptotic expansion of $\mathcal{J}_{k}(t)$ for $k>-\frac{1}{2}$, we have

$$
\mathcal{J}_{k}(r) \simeq r^{-\frac{1}{2}}\left[A_{1}(r) \cos \left(r-\frac{2 k+1}{4} \pi\right)+A_{2}(r) \sin \left(r-\frac{2 k+1}{4} \pi\right)\right]
$$

for large $r$ and

$$
A_{1}(r)=\sum_{\ell=0}^{\infty} c_{1}(\ell) r^{-2 \ell}, A_{2}(r)=\sum_{\ell=0}^{\infty} c_{2}(\ell) r^{-2 \ell-1},
$$

for some explicit coefficients $c_{\sigma}(\ell), \sigma=1,2$. As a consequence, we may write

$$
\tilde{a}_{j}(\tau, \xi)=\sqrt{2 \pi} 2^{-\frac{j}{2}} \cdot 2^{j} \tilde{\chi}_{0}(\tau) \sum_{ \pm} \psi_{\varpi}^{ \pm}(\xi) e^{\mp i \frac{2 \varpi+1}{4} \pi} \int \tilde{\tilde{\chi}}(s) \cdot e^{-2 \pi i 2^{j} s(\tau \mp|\xi|)} d s
$$

where $\psi_{\varpi}^{ \pm}(\xi)=\varphi(\xi) \mathcal{A}_{ \pm}\left(2 \pi 2^{j} \xi\right)|\xi|^{-\varpi-\frac{1}{2}}$ and

$$
\left\{\begin{aligned}
\tilde{\chi}_{0}(\tau) & =|\tau|^{\beta} \chi_{0}(\tau) \\
\tilde{\tilde{\chi}}(s) & =\tilde{\chi}(s) s^{-\frac{1}{2}}
\end{aligned}\right.
$$

Here, $\mathcal{A}_{ \pm}(\xi)=\left(A_{1}(|\xi|) \mp i A_{2}(|\xi|)\right) / 2$ belongs to the classical symbol of order zero $S_{1,0}^{0}\left(\mathbb{R}^{n}\right)$.

Since the function is localized in the high frequency, we now involve the asymptotic expansion for Bessel functions which leads to the half wave operator. Write

$$
\mathscr{F}_{j}(f)(x, t)=\mathscr{F}_{j}^{+}(f)(x, t)+\mathscr{F}_{j}^{-}(f)(x, t),
$$

where

$$
\begin{aligned}
\mathscr{F}_{j}^{ \pm}(f)(x, t) & =2^{-j\left(\varpi-\beta+\frac{1}{2}\right)} e^{\mp i \frac{2 \varpi+1}{4} \pi} \iint e^{2 \pi i 2^{j}(x \cdot \xi+t \tau)} \mathscr{A}_{ \pm}(\tau, \xi) \widehat{f}_{j}(\xi) d \xi d \tau, \\
\mathscr{A}_{ \pm}(\tau, \xi) & =2^{j} \tilde{\chi}_{0}(\tau) \psi_{\varpi}^{ \pm}(\xi) \int \tilde{\tilde{\chi}}(s) \cdot e^{-2 \pi i 2^{j} s(\tau \mp|\xi|)} d s .
\end{aligned}
$$

It is not hard to see

$$
\begin{aligned}
\mathscr{F}_{j}^{ \pm}(f)(x, t)= & 2^{-j\left(\varpi-\beta+\frac{1}{2}\right)} e^{\mp i \frac{2 \varpi+1}{4} \pi} 2^{j} \\
& \times \iint e^{2 \pi i 2^{j} x \cdot \xi} \psi_{\varpi}^{ \pm}(\xi) \widehat{\tilde{\chi} 0}\left(2^{j}(s-t)\right) \tilde{\tilde{\chi}}(s) \widehat{f_{j}}(\xi) e^{ \pm 2 \pi i 2^{j}|\xi| s} d \xi d s \\
= & 2^{-j\left(\varpi-\beta+\frac{1}{2}\right)} e^{\mp i \frac{2 \varpi+1}{4} \pi} 2^{j} \\
& \times \iint e^{2 \pi i x \cdot \xi} \psi_{\varpi}^{ \pm}\left(2^{-j} \xi\right) \widehat{\tilde{\chi}_{0}}\left(2^{j}(s-t)\right) \tilde{\tilde{\chi}}(s) \widehat{f}(\xi) e^{ \pm 2 \pi i|\xi| s} d \xi d s \\
= & 2^{-j\left(\varpi-\beta+\frac{1}{2}\right)} e^{\mp i \frac{2 \varpi+1}{4} \pi} 2^{j} \\
& \times \iint \tilde{\tilde{\chi}}(s) e^{ \pm i s \sqrt{-\Delta}} \tilde{\Delta}_{j} f(y) \widehat{\tilde{\chi}_{0}}\left(2^{j}(s-t)\right) \check{\psi}_{\varpi}^{ \pm}\left(2^{j}(x-y)\right) 2^{j n} d y d s .
\end{aligned}
$$

Applying Young's inequality to the above expressions, we have $\left\|\mathscr{F}_{j}^{ \pm}(f)\right\|_{L^{p}\left(\mathbb{R}^{n} \times \mathbb{R}\right)} \leq 2^{-j\left(\varpi-\beta+\frac{1}{2}\right)}\left\|\check{\psi}_{\varpi}^{ \pm}\right\|_{L^{1}\left(\mathbb{R}^{n}\right)}\left\|\widehat{\tilde{\chi_{0}}}\right\|_{L^{1}(\mathbb{R})}\left\|\tilde{\tilde{\chi}}(s) u_{j}^{ \pm}(s, y)\right\|_{L^{p}\left(\mathbb{R}^{n} \times \mathbb{R}\right)}$, 
where $u_{j}^{ \pm}(s, y):=e^{ \pm i s \sqrt{-\Delta}} \tilde{\Delta}_{j} f(y)$.

At this stage, we use local smoothing estimate for wave equations with $p>2$ to get

$$
\left\|\mathscr{F}_{j}^{ \pm}(f)\right\|_{L^{p}\left(\mathbb{R}^{n} \times \mathbb{R}\right)} \leq C 2^{j \mu}\|f\|_{L^{p}\left(\mathbb{R}^{n}\right)},
$$

where $\mu=-\varpi+\beta-\frac{1}{2}+\gamma, \forall \gamma>\gamma(p, n)$. In view of (2.13), we have under the same conditions

$$
\left\|\mathscr{F}_{j}(f)\right\|_{L^{p}\left(\mathbb{R}^{n} \times \mathbb{R}\right)} \leq C 2^{j \mu}\|f\|_{L^{p}\left(\mathbb{R}^{n}\right)} .
$$

Summing up all the estimates on $\mathscr{F}_{j}(f)(x, t)$ by means of Littlewood-Pelay's theory, we have in view of (2.7) and (2.10)

$$
\left\|\left|\partial_{t}\right|^{\beta}\left(\chi(t) \mathscr{M}_{t}^{\alpha}(f)\right)\right\|_{L^{p}\left(\mathbb{R}^{n} \times \mathbb{R}\right)} \leq C \sum_{j \geq 0} 2^{\mu j}\|f\|_{p}+C_{N}^{\prime} 2^{-\frac{N}{2}}\|f\|_{p},
$$

for some suitable constant $C>0$. To ensure the geometric series converge, we need

$$
\mu<0, \gamma>\gamma(p, n)
$$

which in turn is equivalent to

$$
\beta<\alpha+\frac{n-1}{2}-\gamma(p, n)
$$

On the other hand, if $\beta>\frac{1}{p}$, we can use Sobolev embedding to obtain

$$
\begin{aligned}
\| \sup _{1<t<2}\left|\mathscr{M}_{t}^{\alpha}(f)(x)\right| & \|_{L^{p}\left(\mathbb{R}^{n}\right)} \\
& \leq C\left\|\left\langle\partial_{t}\right\rangle^{\beta}\left(\chi(t) \mathscr{M}_{t}^{\alpha}(f)\right)\right\|_{L^{p}\left(\mathbb{R}^{n} \times \mathbb{R}\right)} \leq C\|f\|_{p},
\end{aligned}
$$

where $\left\langle\partial_{t}\right\rangle=\left(1-\partial_{t}^{2}\right)^{\frac{1}{2}}$. The conditions for the exponents $\mu, \alpha, \beta$ are summarized to

$$
\alpha>-\varepsilon(p, n) .
$$

Step 4. In this step, we reduce the general situation when $t>0$ to the particular case of $t \in[1,2]$. To achieve this, we will use Littlewood-Paley theory by writing

$$
\mathscr{M}_{t}^{\alpha} f(x)=\sum_{j=0}^{\infty} \mathscr{M}_{j, t}^{\alpha} f(x)
$$

where

$$
\mathscr{M}_{j, t}^{\alpha} f(x)=\int e^{2 \pi i x \cdot \xi} \varphi_{j}(t \xi) \widehat{m}_{\alpha}(t \xi) \widehat{f}(\xi) d \xi
$$

with $\varphi_{j}(\xi)$ defined at the beginning of the proof. Thus it suffices to see for $\alpha$ and $p$ satisfying (2.15), there exists some $\mu<0$ such that

$$
\left\|\sup _{t>0}\left|\mathscr{M}_{j, t}^{\alpha} f(x)\right|\right\|_{L^{p}\left(\mathbb{R}^{n}\right)} \leq C \cdot 2^{j \mu}\|f\|_{L^{p}} .
$$


If $j>0$, we may now reduce (2.16) to (2.14). Denote by $\widehat{\dot{\Delta}_{\ell} f}(\xi)=$ $\varphi\left(2^{-\ell}|\xi|\right) \widehat{f}(\xi)$ for all $\ell \in \mathbb{Z}$ and notice that we have

$$
\mathscr{M}_{j, t}^{\alpha} f(x)=\sum_{|\ell| \leq 100} \mathscr{M}_{j, t}^{\alpha}\left(\dot{\Delta}_{j+k+\ell} f\right)(x),
$$

whenever $2^{-k} \leq t \leq 2^{-k+1}$ for $k \in \mathbb{Z}$, and the following pointwise estimation

$$
\sup _{t>0}\left|\mathscr{M}_{j, t}^{\alpha} f(x)\right| \leq\left(\sum_{k \in \mathbb{Z}^{2}} \sup _{2^{-k} \leq t \leq 2^{-k+1}}\left|\mathscr{M}_{j, t}^{\alpha} f(x)\right|^{p}\right)^{\frac{1}{p}} .
$$

Now, we claim that

$$
\left\|\sup _{t \in\left[2^{-k}, 2^{-k+1}\right]}\left|\mathscr{M}_{j, t}^{\alpha} f(x)\right|\right\|_{p} \leq C \cdot 2^{\mu j}\|f\|_{p}, \forall k \in \mathbb{Z} .
$$

Under this claim, the left side of (2.16) can be estimated as

$$
\begin{aligned}
& \left(\sum_{k \in \mathbb{Z}}\left\|\sup _{t \in\left[2^{-k}, 2^{-k+1}\right]} \mathscr{M}_{j, t}^{\alpha}\left(\sum_{|\ell| \leq 100} \dot{\Delta}_{j+k+\ell} f\right)(x)\right\|_{p}^{p}\right)^{\frac{1}{p}} \\
\leq & C \cdot 2^{\mu j}\left(\sum_{k \in \mathbb{Z}}\left\|\sum_{|\ell| \leq 100} \dot{\Delta}_{j+k+\ell} f(x)\right\|_{p}^{p}\right)^{\frac{1}{p}} \\
\leq & C^{\prime} \cdot 2^{\mu j}\left\|\left(\sum_{k \in \mathbb{Z}}\left|\dot{\Delta}_{k} f(x)\right|^{2}\right)^{\frac{1}{2}}\right\|_{p} .
\end{aligned}
$$

Invoking the standard square-function inequality, we see the last term is clearly bounded by $2^{\mu j}\|f\|_{p}$, where $2<p<\infty$.

To show (2.17), we start with a standard scaling to get

$$
\left\|\sup _{t \in\left[2^{-k}, 2^{-k+1}\right]}\left|\mathscr{M}_{j, t}^{\alpha} f(x)\right|\right\|_{p} \leq\left\|\sup _{t \in[1,2]}\left|\mathscr{M}_{j, t}^{\alpha}\left(f_{2^{k}}\right)\left(2^{k} x\right)\right|\right\|_{p},
$$

where $f_{2^{k}}(x)=f\left(2^{-k} x\right)$. In view of the following formula,

$$
\widehat{\mathscr{M}_{j, t}^{\alpha} f}(\xi)=\widehat{m}_{\alpha}(t \xi) \varphi_{j}(\xi) \widehat{f}(\xi)+\int_{0}^{t} \widehat{m}_{\alpha}(t \xi)\left\langle\xi, \nabla \varphi_{j}(\theta \xi)\right\rangle \widehat{f}(\xi) d \theta,
$$

we have

$$
\sup _{1<t<2}\left|\mathscr{M}_{j, t}^{\alpha} f(x)\right| \leq \sup _{t \in[1,2]}\left|\mathscr{M}_{t}^{\alpha}\left(\Delta_{j} f\right)(x)\right|+\int_{1}^{2} \sup _{t \in[1,2]}\left|\mathscr{M}_{t}^{\alpha}\left(\tilde{\Delta}_{j}^{\theta} f\right)(x)\right| d \theta,
$$

where

$$
\widehat{\tilde{\Delta}}_{j}^{\theta} f(\xi):=\left\langle\xi, \nabla \varphi_{j}(\theta \xi)\right\rangle \widehat{f}(\xi) .
$$

The argument in Step 3 works to $\sup _{t \in[1,2]}\left|\mathscr{M}_{t}^{\alpha}\left(\tilde{\Delta}_{j}^{\theta} f\right)(x)\right|$ as well, yielding an appropriate upper bound independent of $\theta \in[1,2]$. Therefore (2.17) follows from Step 3 and rescaling. 
It remains to handle the case when $j=0$. This is standard as we denote $\widehat{\Phi}(\xi)=\varphi_{0}(\xi) \widehat{m}_{\alpha}(\xi)$, then $\int \Phi(x) d x=\widehat{m}_{\alpha}(0)$ and

$$
\mathscr{M}_{0, t}^{\alpha} f(x, t)=\Phi_{t} * f(x),
$$

where $\Phi_{t}(x)=t^{-n} \Phi\left(x t^{-1}\right)$. Hence

$$
\sup _{t>0}\left|\mathscr{M}_{0, t}^{\alpha} f(x, t)\right| \leq C M_{H L}(f)(x)
$$

and the $L^{p}$ estimate follows.

Finally, notice that the above arguments also work when $\alpha \in \mathbb{C}$ and Re $\alpha>-\varepsilon(p, n)$. We complete the proof of Theorem 1.1 and this extends Stein's result for $n \geq 2$ and $p>2$.

Remark 2.1. If one asks the same question for general hypersurface rather than standard sphere, for example, we replace $|x|=\sqrt{x_{1}^{2}+\cdots x_{n}^{2}}$ by another norm

$$
\|x\|_{s}=\left(x_{1}^{s}+\cdots+x_{n}^{s}\right)^{\frac{1}{s}}, 0<s<\infty,
$$

in the definition of $m_{\alpha}(x)$ with $\alpha \geq 0$, the above results deduced above fails to hold. In fact, it is shown in [6] that if one considers the surfaces where the Gaussian curvature is allowed to vanish, the $L^{p}$ exponents for the corresponding maximal operators are often worse. We refer to [6] for those cases.

\section{FURTHER DISCUSSIONS}

At the end of this paper, we discuss on some directions which might be helpful to further studies. It seems interesting to study the following problems.

(1). Is the relation $\operatorname{Re} \alpha>-\varepsilon(p, n)$ implied by local smoothing estimate optimal for (1.6) to hold ?

(2). The researches concerning Stein's maximal spherical operators appear in the literature focusing mainly on two aspects. One is to study the variable coefficient version of the maximal functions when $\alpha=0$, as can be found in [15]. The other one is to extend the relation between $\alpha$ and $p$ in Theorem 1.1 for classical maximal operator (1.5). A natural question is how to combine these two directions by establishing the variable coefficient version of the analytic family of spherical means.

(3). It is well known that Stein's $L^{p}-L^{p}$ bounds on maximal spherical means can be generalized to certain $L^{q}-L^{p}$ inequalities, see Schlag [11, 12]. If $\alpha=0$, there is a variable coefficient version of Schlag's $L^{q}-L^{p}$ estimates in [13]. The question is whether this is valid for $\alpha \neq 0$.

(4). When $\alpha=0$, it is shown that $p>\frac{n}{n-1}$ is necessary for (1.6). It is interesting to know if the weak type $(p, p)$ estimate holds at the end-point $p=\frac{n}{n-1}$. At this stage, classical Calderón-Zygmund decomposition may be useful. 
(5). It seems also interesting to know whether it is possible to prove the maximal inequality for extended exponents in Theorem 1.1 without using local smoothing estimate. If this is true, it will be an evidence to support the likely true local smoothing conjecture.

\section{Appendix A.}

For the convenience of reading, we give the proof of two facts cited in Section 1.

A.1. The proof of (1.2). Using polar coordinates, we can do the following calculation

$$
\begin{aligned}
\widehat{m}_{\alpha}(\xi) & =\int_{\mathbb{R}^{n}} e^{-2 \pi x \cdot \xi} m_{\alpha}(x) d x \\
& =\frac{1}{\Gamma(\alpha)} \int_{\mathbb{R}^{n}} e^{-2 \pi x \cdot \xi}\left(1-|x|^{2}\right)_{+}^{\alpha-1} d x \\
& =\frac{1}{\Gamma(\alpha)} \int_{0}^{1}\left(1-\rho^{2}\right)^{\alpha-1} \rho^{n-1} \int_{\mathbb{S}^{n-1}} e^{-2 \pi \rho w \cdot \xi} d \sigma(w) d \rho \\
& =\frac{2 \pi}{\Gamma(\alpha)} \int_{0}^{1}\left(1-\rho^{2}\right)^{\alpha-1} \rho^{\frac{n-2}{2}+1} J_{\frac{n-2}{2}}(2 \pi|\xi| \rho) d \rho \\
& =\pi^{-\alpha+1}|\xi|^{-\frac{n}{2}-\alpha+1} \mathcal{J}_{\frac{n}{2}+\alpha-1}(2 \pi|\xi|),
\end{aligned}
$$

where we have used the following two identities (see Appendix B in [5])

$$
\begin{aligned}
\int_{\mathbb{S}^{n-1}} e^{-2 \pi \theta \cdot \xi} d \theta & =\frac{2 \pi}{|\xi|^{\frac{n-2}{2}}} J_{\frac{n-2}{2}}(2 \pi|\xi|), \\
\int_{0}^{1} J_{\mu}(t s) s^{\mu+1}\left(1-s^{2}\right)^{\nu} d s & =\frac{\Gamma(\nu+1) 2^{\nu}}{t^{\nu+1}} J_{\mu+\nu+1}(t) .
\end{aligned}
$$

A.2. The proof of (1.16). Here we give a new proof of (1.16). It is based on the following fact

$$
J_{\frac{1}{2}}(r)=\frac{\sqrt{r}}{\sqrt{2} \Gamma(1 / 2)} \int_{-1}^{1} e^{i s r} d s=\frac{1}{\sqrt{2} \Gamma(1 / 2)} \frac{\sin (r)}{\sqrt{r}} .
$$

Letting $\alpha=\frac{3-n}{2}$, we obtain

$$
\begin{aligned}
t \mathcal{F}_{x \rightarrow \xi}\left(M_{t}^{\alpha}(f)(\cdot)\right)(\xi) & =t \hat{m}_{\alpha}(t \xi) \hat{f}(\xi) \\
& =t \frac{\pi^{\frac{n-1}{2}}}{|t \xi|^{\frac{1}{2}}} J_{\frac{1}{2}}(2 \pi|t \xi|) \hat{f}(\xi) \\
& =\tilde{c}_{n} \frac{\sin (t \xi)}{|\xi|} \hat{f}(\xi) \\
& =\tilde{c}_{n} \mathcal{F}_{x \rightarrow \xi}\left(\frac{\sin (t \sqrt{-\Delta})}{\sqrt{-\Delta}} f\right)(\xi) .
\end{aligned}
$$


After taking inverse Fourier transform, we have (1.16) solves the Cauchy problem (1.10).

\section{REFERENCES}

[1] J. Bourgain, On the spherical maximal function in the plane. IHES, 1985.

[2] J. Bourgain, Averages in the plane over convex curves and maximal operators. J d'analyse Math. 47. (1986), 69-85.

[3] J. Bourgain and C. Demeter, The proof of the $\ell^{2}$ decoupling conjecture. Annals of Math. (2) 182 (2015), no. 1, 351-389.

[4] G. Garrigós, W. Schlag and A. Seeger, Improvements in Wolff's inequality for decompositions of cone multipliers. Unpublished preprint, available online (2008).

[5] L. Grafakos, Classical Fourier Analysis. Second Edition, Springer, 2008.

[6] A. Iosevich and E. Sawyer, Maximal averages over surfaces. Adv. Math. 132 (1997), no.1, 46-119

[7] L. Kolasa and T. Wolff, On some variants of the Kakeya problem. Pacific. J. Math. 190 (1999),111-154.

[8] I. Łaba and T. Wolff, A local smoothing estimate in higer dimensions. J. d'analyse Math., 88(2002), 149-171.

[9] G. Mockenhaupt, A. Seeger and C. D. Sogge, Wavefront sets, local smoothing and Bourgain's circular maximal theorem. Ann. Math.,136 (1992), 207-218.

[10] G. Mockenhaupt, A. Seeger and C. D. Sogge, Local smoothing of Fourier integral operators and Carleson-Sjölin estimates. J. Amer. Math. Soc., 6(1993), 65-130.

[11] W. Schlag, $L^{p}-L^{q}$ estimates for the circular maximal function, Ph.D. Thesis. Californian Institute of Technology,1996.

[12] W. Schlag, A generalization of Bourgain's circular maximal theorem. Journal Amer. Math. Soc. 10(1997), 103-122.

[13] W. Schlag and C. D. Sogge Local smoothing estimates related to the circular maximal theorem. Math. Reaserch Letters, 4(1997), 1-15.

[14] C. D. Sogge, Propogation of singularity and maximal functions in the plane. Invent. Math., 104(1991), 349-376.

[15] C. D. Sogge, Fourier integrals in classical analysis. Cambridge Univ. Press,1993.

[16] E. M. Stein, Maximal functions; spherical means. Proc. Nat. Acad. Sc. U.S.A., 73 (1976), 2174-2175.

[17] E. M. Stein, Harmonic analysis, real vairable methods, orthogonality and oscillatory integrals. Princeton Univ. Press (1993).

[18] E. M. Stein and G. Weiss Introduction to Fourier analysis on Euclidean spaces. (1971), Princeton Univ. Press. Princeton, N.J.

[19] G. N. Watson, A treatise on the theory of Bessel function. Second Edition, Cambridge University Press, 1944.

[20] T. Wolff, Local smoothing type estimates on $L^{p}$ for large $p$. Geom. Funct. Anal., 10 (2000), 1237-1288.

Institute of Applied Physics and Computational Mathematics, Beijing 100088, CHINA

E-mail address: miao_changxing@aliyun.com , miao_changxing@iapcm.ac.cn

LAGA(UmR 7539), Institut Galilée, Université Paris 13, Sorbonne Paris Cité, France, Beijing International Center for Mathematical Research, Peking University, BeiJing 100871, China

E-mail address: geewey_young@pku.edu.cn 
Université de Nice - Sophia Antipolis, Laboratoire J. A. Dieudonné, 06108 Nice Cedex 02, France

E-mail address: zhengjiqiang@gmail.com 